\title{
Quelle attitude adopter face aux compensations
}

\author{
Bruno Henggi ${ }^{a}$, Charlotte Schweizer ${ }^{b}$ \\ a responsable Affaires publiques de la $\mathrm{FMH} ;{ }^{b}$ cheffe de la division Communication
}

Les nouvelles prescriptions sur la manière de gérer les produits thérapeutiques qui entrent en vigueur le $1^{\text {er }}$ janvier 2020 comportent des règles plus strictes et ont un impact direct sur le travail quotidien des médecins. Elles sont définies dans l'ordonnance sur l'intégrité et la transparence dans le domaine des produits thérapeutiques (OITPTh), qui vise à renforcer l'intégrité et à augmenter la transparence dans la manière de gérer les produits thérapeutiques. Elle règle donc en détail les rabais et compensations de la part des fournisseurs de médicaments. De plus, elle comprend des dispositions relatives aux dons destinés à la recherche et à la formation postgraduée et continue. Elle prévoit également des règles strictes pour ce qui concerne l'acceptation de cadeaux et invitations à des repas.

Pour les médecins, cela signifie se familiariser avec les dispositions de la nouvelle ordonnance afin de pouvoir les respecter et les appliquer correctement mais aussi documenter leurs actes de manière systématique et complète. En tant qu'association professionnelle, la FMH considère qu'il est de son devoir de rendre ses membres attentifs à cette réglementation plus stricte et de les informer plus largement des nouvelles dispositions légales. Nous comptons le faire dans le cadre d'une série en quatre volets.

Dans le présent article, nous nous intéressons de plus près aux questions se posant aux médecins dans le contexte des compensations accordées en contrepartie de prestations équivalentes. Nous les traitons à l'aide d'exemples pratiques tels qu'ils peuvent survenir dans le quotidien des médecins.

\section{Compensations accordées en contrepartie de prestations équivalentes}

Question: Que comprennent les compensations accordées en contrepartie de prestations équivalentes?

Réponse: Pour qu'une compensation soit admise conformément à la nouvelle ordonnance, le professionnel de santé (p. ex. médecin) doit fournir une prestation équivalente en contrepartie. Lors de l'achat de médicaments soumis à ordonnance, cette contrepartie peut par exemple consister à prendre en charge la logistique, les frais de stockage ou le risque de gestion des stocks. Les frais supportés peuvent alors être compensés sous la forme d'un rabais sur la quantité. De même, les activités d'enseignement, d'expertise ou de conseil, ou encore l'exécution d'études scientifiques ou cliniques, peuvent représenter une contrepartie adéquate et donc admise. C'est aussi le cas des comptes rendus d'expériences pratiques, lorsqu'ils sont publiés dans un organe de presse spécialisée reconnu. Enfin, la collaboration au sein d'organes consultatifs et la participation à des ateliers ou à des études de marché peuvent aussi donner lieu à une compensation, à condition qu'ils ne soient pas organisés à des fins publicitaires.

Dans tous les cas, les compensations accordées en contrepartie de prestations équivalentes doivent, premièrement, faire l'objet d'une convention écrite indiquant la nature et l'ampleur de la prestation et de la compensation, et deuxièmement, être proportionnées à ladite prestation.

Il est également important de savoir que les prestations que le professionnel fournit pour lui-même ou pour s'acquitter d'obligations légales, ou pour lesquelles il est déjà rémunéré d'une autre manière, ne peuvent pas être indemnisées par une compensation supplémentaire. 
La prise en charge de frais de repas dans le cadre d'un entretien professionnel n'a pas besoin de faire l'objet d'une convention écrite si le montant ne dépasse pas 100 francs. Si le montant est supérieur, une convention écrite est alors nécessaire. Ici aussi, la prestation fournie en contrepartie par le professionnel devra être proportionnée aux frais de repas. Il ne doit pas s'agir d'une simple invitation à un repas d'affaires.

\section{Equivalence de la compensation}

Question: Comment déterminer si une compensation est équivalente à la prestation fournie en contrepartie? Réponse: Il n'est pas possible de répondre de manière générale à cette question. C'est avant tout le bon sens qui prime. Il est important que le rapport entre contreprestation et indemnisation soit adapté. Si la compensation sort de ce cadre, il faudrait y renoncer. En cas de doute, il est possible de demander à l'entreprise pharmaceutique ou à la personne responsable de confirmer cette équivalence. En l'absence de confirmation, il faudrait envisager, par sécurité, de refuser l'indemnisation en question.

\section{Commandes en ligne}

Question: Une entreprise décide de ne plus accorder de rabais mais de verser $1 \%$ aux médecins lors de commandes en ligne à titre de contreprestation. Cette réduction est-elle admissible?

Réponse: Une remise de $1 \%$ peut être considérée comme une compensation (de la part du fournisseur de médicaments) pour une contreprestation (fournie par le médecin), en raison d'un processus de commande simplifié. Pour être admises, les indemnisations de prestations doivent reposer sur une convention écrite et être proportionnées à la prestation en question. Dans ce cas, nous conseillons aux médecins de conclure avec l'entreprise une convention-cadre écrite confirmant l'équivalence de l'indemnisation et de la prestation et décrivant les modalités de cette dernière (commande en ligne). Si ces conditions sont remplies, ce type d'indemnisation est admis pour compenser la prestation fournie en contrepartie par le médecin.

Si l'entreprise refuse de confirmer l'équivalence entre la prestation et l'indemnisation ou si aucune convention écrite n'est conclue, on est alors en présence non pas d'une compensation mais d'un rabais. Dans ce cas, nous recommandons aux médecins de répercuter ce dernier directement sur les patients ou de payer la totalité du prix.

\section{Escompte}

Question: Lors d'une commande de médicaments, le professionnel de santé a la possibilité de bénéficier d'un escompte de $3 \%$ s'il paie la facture dans les 10 jours. Les médecins ont-ils le droit d'accepter une telle remise?

Réponse: Un escompte, et plus exactement un paiement anticipé, représente en principe une contreprestation: il permet à l'entreprise bénéficiaire de disposer de liquidités et de réduire le risque de recouvrement. A partir du $1^{\text {er }}$ janvier 2020, la loi prévoit toutefois la conclusion d'une convention écrite pour les compensations accordées en contrepartie de prestations équivalentes. De plus, pour être admise, la compensation doit être équivalente à la contreprestation. La question se pose donc ici de savoir si ces 3\% entrent encore dans ce cadre. Il reviendra aux tribunaux de trancher quant à la hauteur des escomptes admis. Par précaution, il ne faudrait pas pour le moment que l'escompte dépasse les $2 \%$.

\section{Base légale de l'OITPTh: explication}

Le but de la nouvelle ordonnance est d'éviter que la prescription, la remise, l'utilisation ou l'achat de médicaments soumis à ordonnance soient influencés de quelque manière que ce soit. Au lieu d'imposer une interdiction totale de toute marque d'attention, la nouvelle ordonnance établit une liste des exceptions qui indique de manière exhaustive ce qui est juridiquement encore possible.

Cela inclut les compensations accordées en contrepartie de prestations équivalentes, en particulier lors de la commande et de la livraison de médicaments. L'équivalence de la contreprestation et de l'indemnisation doit être inscrite dans une convention écrite. De plus, les modalités doivent être décrites aussi précisément que possible et de manière à pouvoir être vérifiées. Pour le moment, faute d'expérience, il est impossible de savoir ce que les autorités considéreront comme des prestations "équivalentes». Elles s'inspireront cependant des dispositions suivantes, qui figurent dans le rapport explicatif:

- L'indemnité doit correspondre à l'ampleur de la prestation fournie et au travail qu'elle a occasionné.

- L'indemnité est versée séparément ou décomptée avec les prestations fournies par le bénéficiaire de la contrepartie.

- Elle doit être moins élevée pour des prestations qui peuvent être préparées ou fournies dans le cadre de l'exercice normal de la profession que pour celles qui nécessitent un travail supplémentaire. 
- Les prestations qui ne nécessitent pas de travail supplémentaire de la part du professionnel, à savoir les prestations qu'il doit de toute façon fournir, sont interdites.

- Les doubles compensations, résultant du fait que la prestation a été ou sera rémunérée d'une autre façon, sont également interdites (p. ex. prestation rémunérée par le salaire ou facturée au client ou à l'assurance).

- Enfin, l'indemnisation de prestations qui profitent aux professionnels eux-mêmes est elle aussi interdite.

La prise en charge de frais de repas par une entreprise pharmaceutique dans le cadre d'un entretien professionnel est exemptée de l'obligation d'établir une convention écrite, à condition que le montant ne dépasse pas 100 francs (boissons comprises).

\section{Art. 7 Compensations accordées en contrepartie de prestations équivalentes}

1 Les compensations au sens de l'art. 55, al. 2, let. c, LPTh accordées à des professionnels ou à des organisations en contrepartie de prestations équivalentes sont admises pour autant qu'elles:

a. se fondent sur une convention écrite indiquant la nature et l'ampleur de la prestation et de la compensation, et qu'elles

b soient proportionnées à la prestation.

2 Dans le cadre d'un entretien professionnel, la prise en charge de frais de repas jusqu'à un maximum de 100 francs est exemptée de l'obligation visée à l'al.1, let. a.
3 Un professionnel ou une organisation ne peut recevoir de compensation en particulier pour les prestations:

a qu'il fournit pour lui-même;

b qu'il fournit en s'acquittant d'obligations légales, ou

c pour lesquelles il est rémunéré d'une autre manière.

4 Les compensations visées à l'al. 1 sont admises en particulier pour:

a des prestations en rapport avec l'achat de médicaments soumis à ordonnance, telles que la prise en charge de la logistique, des frais de stockage ou du risque de gestion des stocks;

b des activités d'enseignement, d'expertise ou de conseil, ou la réalisation d'études scientifiques et d'essais cliniques;

c des rapports d'expérience pratique publiés dans un média scientifique reconnu s'adressant aux professionnels;

d la participation à des comités consultatifs, à des ateliers ou à des études de marché, pour autant qu'ils ne soient pas à but publicitaire.

A titre de précaution, nous recommandons aux médecins de prendre certaines mesures, à savoir:

se faire confirmer par écrit l'équivalence de la prestation et de I'indemnisation;

- en l'absence de confirmation écrite, répercuter l'indemnisation.

\section{\{FMH}
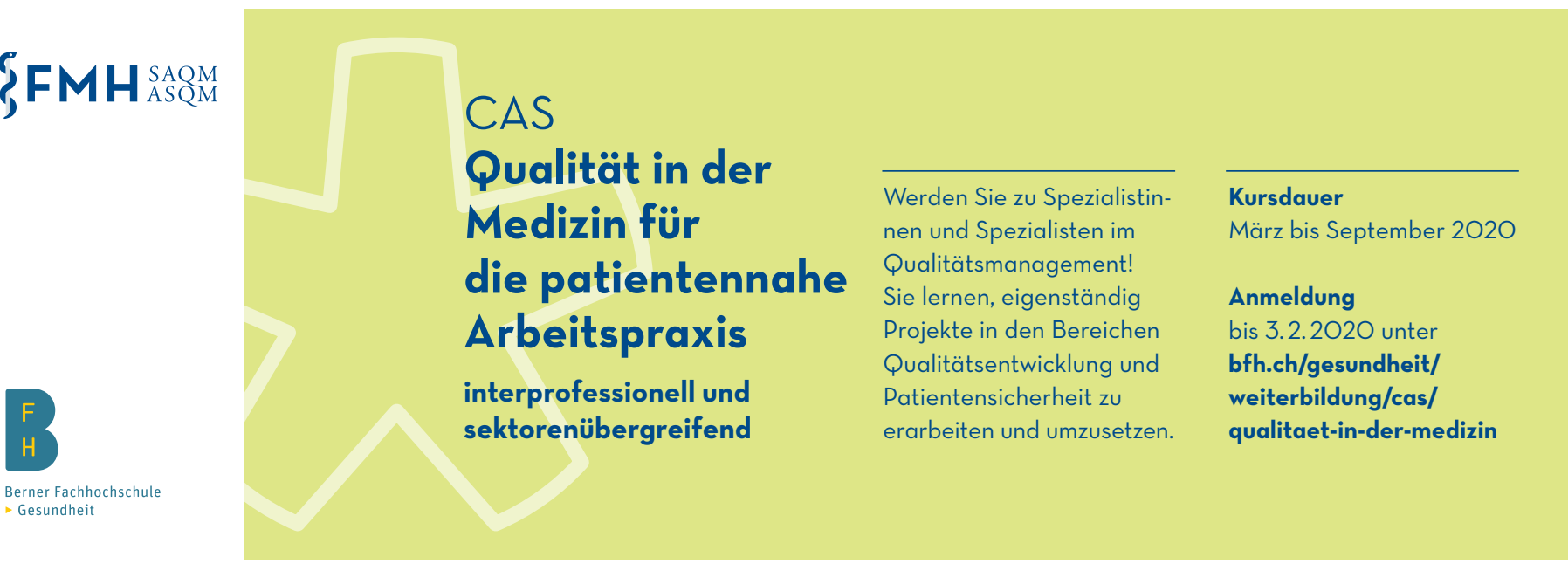